\title{
Ocular Morbidity, Clinical Profile and Visual Loss in Vitamin-D Deficiency; A Systemic Correlation
}

\section{Meera Mohanakumar ${ }^{1}$, Radha Annamalai ${ }^{2 *}$, Shriraam Mahadevan $^{3}$ and M Muthayya ${ }^{2}$}

${ }^{1}$ Post Graduate Resident, Department of Ophthalmology, Sri Ramachandra Institute of Higher Education and Research, Chennai, India

${ }^{2}$ Professor of Ophthalmology, Department of Ophthalmology, Sri Ramachandra Institute of Higher Education and Research, Chennai, India

${ }^{3}$ Professor of Endocrinology, Department of Endocrinology, Sri Ramachandra

Institute of Higher Education and Research, Chennai, India

*Corresponding Author: Radha Annamalai, Professor of Ophthalmology,

Department of Ophthalmology, Sri Ramachandra Institute of Higher Education and Research, Chennai, India.
Received: March 07, 2020

Published: April 30, 2020

(C) All rights are reserved by Radha

Annamalai., et al.

\section{Abstract}

Purpose: To determine the spectrum of ocular manifestations of vitamin-D deficiency/insufficiency, its effects on vision and the levels at which they occur.

Materials and Methods: A prospective study done in concurrence with the department of endocrinology over a period of 1 year on 150 patients who had been referred to the ophthalmology clinic for screening and treatment of ocular complaints.

Results: Dry eye disease(DED) was found in 102 patients (78\%), followed by glaucoma in 33 (27\%) patients, diabetic retinopathy in $30(22 \%)$, myopia as the common refractive error in 25 patients (16.7\%), hypertensive retinopathy in 13 out of 150 (8.7\%) and age related macular degeneration(ARMD) in 6 patients (4\%).

Among the various ocular features, keratoconjunctivitis sicca was the most common manifestation across all age groups with vitamin-D deficiency; followed by glaucoma.

In those with dry eye, decreased TBUT occurred in 30 patients and decreased Schirmer's in 72 patients. This was found to be statistically significant with a p-value of 0.381 . Among those with glaucoma, 4 (2.7\%) were disc suspects, $2(1.3 \%)$ had primary angle closure and $13(8.3 \%)$ patients had primary open angle glaucoma and 11 had ocular hypertension. The incidence of glaucoma was higher in women with deficiency and this was found to be statistically significant with $\mathrm{p}=0.03$. Myopia in $16.7 \%$ and diabetic retinopathy in $22 \%$ were other features. Visual loss and visual field defects occurred predominantly in patients with glaucoma due to optic nerve head changes.

Conclusion: Vitamin-D levels and genetic variations influence the development of a wide range of ocular pathologies, such as myopia, age-related macular degeneration, diabetic retinopathy, glaucoma and uveitis with its anti-angiogenic, anti-inflammatory and antifibrotic properties. Many tissues in the eye activate and respond to vitamin-D, suggesting that vitamin-D is a biologically relevant entity to study throughout the eye.

Keywords: 25-Hydroxy Vitamin-D3; Intraocular Pressure; Tear Breakup Time; Dry Eye Disease; Glaucoma

\section{Abbreviations}

IOP: Intraocular Pressure; TBUT: Tear Breakup Time; DED: Dry Eye Disease; PEE: Punctate Epithelial Erosions; NPDR: Non-Proliferative Diabetic Retinopathy; PDR: Proliferative Diabetic Retinopathy; CSME: Clinically Significant Macular Oedema; ARMD: Age Related Macular Degeneration; POAG: Primary Open Angle Glaucoma; PACG: Primary Angle Closure Glaucoma; NRR: Neuro-Retinal RIM; RNFL: Retinal Nerve Fibre Layer

\section{Introduction}

Vitamin-D, a circulating steroid hormone with multiple functions, has anti-angiogenic, anti-inflammatory and anti-fibrotic properties. An inverse relationship exists between vitamin-D levels and chronic inflammatory conditions. Multiple inhibitory effects are mainly mediated through Vitamin-D receptors (VDR) which has direct effect on gene expression [1]. 
In the eye, vitamin-D target cells were first identified by the presence of vitamin-D dependent calcium binding protein, or calbindin, which was shown to be expressed throughout the human retina. Immunohistochemical staining identified these receptors in the epithelium and endothelium of the cornea, lens, ciliary body, retinal pigment epithelium and ganglion cell layer and retinal photoreceptors [2-4]. Recent research has shown several other actions of vitamin-D in tissues such as skin, smooth muscle, breast, and most recently of VDR and 1-alpha-hydroxylase enzyme in the eye [5]. Vitamin-D is known to be produced in the skin and is hydroxylated in the liver and kidneys for further use by the intestinal epithelium to increase the uptake of dietary calcium.

The primary function of vitamin-D is to help in promotion of calcium absorption and inadequate levels can promote ocular inflammation and degeneration. Vision threatening complications secondary to ophthalmic disease can arise in chronic deficiency. We studied the role of vitamin-D levels in ocular disease, analysed the spectrum of features causing visual loss with relation to the level of deficiency. To our knowledge the affliction on the eye and ocular morbidity with correlation to levels of vitamin-D has never been reported.

\section{Aim of the Study}

The aim of the study is to determine the spectrum of ocular manifestations of vitamin-D deficiency/insufficiency, its effects on vision and the levels at which they occur.

\section{Materials and Methods}

This study was conducted at SRIHER after approval from the institutional ethics committee CSP - MED/19/JUL/54/97. It was a prospective study done in concurrence with the departments of endocrinology over a period of 1 year on 150 patients who had been referred to the ophthalmology clinic for screening and treatment of ocular complaints.

\section{Inclusion criteria}

Patients (males and females) with vitamin-D insufficiency (25$\mathrm{OH}$ vitaminD3 levels between 20 - 29 nanograms/millilitre and vitamin-D deficiency (Mild 10 - 19 nanograms/millilitre, Moderate to Severe $<9$ nanograms/millilitre and Severe $<5$ nanograms $/$ millilitre) above 18 years of age, coming for routine ophthalmic evaluation.

\section{Exclusion criteria}

Patients with known bone metabolism disorders, hyperparathyroidism and those on prior vitamin-D supplementation therapy were not included in the study.

\section{Method}

A detailed clinical history inclusive of systemic, family and drug history was recorded and complete ophthalmologic examination performed in all the study patients.

Venous blood samples ( $\leq 5 \mathrm{~mL}$ ) for determination of serum 25$\mathrm{OH}$ vitaminD3 levels from routine blood samples for other investigations as part of their normal health screening programme was collected for all 150 patients. Patients were categorised as those with vitamin-D insufficiency with $25-\mathrm{OH}$ vitaminD3 levels between 20 - 29 nanograms/millilitre and vitamin-D deficiency when levels were less than 10 nanograms/millilitre. Those with deficiency, were further classified as mild (10-19 nanograms/millilitre), moderate ( $<9$ nanograms/millilitre) and severe when levels were less than 5 nanograms/millilitre).

Ophthalmologic examination commencing with visual acuity testing by the subjective method using standard Snellen's chart according to the international tables and the best corrected visual acuity was calculated. Anterior segment examination using a slitlamp, ophthalmoscopy was done followed by intraocular pressure measurement on a Goldmann's applanation tonometer (in mmHg). In glaucoma suspects, gonioscopy for angle examination with a four mirror Sussman lens and automated perimetry was done.

Tear Breakup Time (TBUT) to detect tear film insufficiency due to evaporative dry eye disease and Schirmer's test for detection of defective secretion was done in all our patients to assess the cause and severity [6]. A TBUT under 10 seconds was considered abnormal according to defined values. Other signs like punctate epithelial erosions (PEE) that stained positively with fluorescein, decreased tear film height, mucous plaques, tear film debris reduced Schirmer's values, staining with Rose Bengal [7] were also looked for and documented.

Following fundus examination by direct and indirect ophthalmoscopy, fundus fluorescein angiography and systemic investigations were performed on those who had retinopathy. FFA (FF 450 Plus, Zeiss India) was done to identify the presence of capillary non perfusion, macular oedema, choroidal neovascular membranes and retinal neovascularization [8].

Pachymetry was performed to assess the central corneal thickness. A Spectral Domain Optical Coherence Tomography (SD-OCT) was done when there was raised intraocular pressures, for retinopathy. OCT (model: Cirrus HD400, Carl Zeiss), a non-invasive three dimensional imaging of the retina, choroid and optic nerve head was done to detect glaucomatous cupping, NRR thinning, retinal oedema, haemorrhage or scarring [9]. 
Investigations were performed during the first visit and during each review. During each follow-up, patients were examined by both the ophthalmologist and the endocrinologist to look for improvement or worsening of both ophthalmic and systemic features.

In all our patients, dry eye was treated with artificial tears, myopia with spectacles or contact lens and glaucoma with anti-glaucoma drugs, laser iridotomy or trabeculectomy. diabetic retinopathy with control of blood sugars and lasers as and when required. Patient's with ARMD were given anti-oxidant tablets and were asked to monitor their vision using home Amsler's chart.

\section{Statistical analysis}

The collected data was analysed with IBM SPSS statistics software 23.0 Version. To describe about the data descriptive statistics frequency analysis, percentage analysis was used for categorical variables and the mean and standard deviation (SD) were used for continuous variables. To find the significant difference between the bivariate samples in independent groups, the Unpaired sample t-test was used. To find the significance in categorical data, ChiSquare test was used. In all our analysis, a probability value of $\mathrm{p}<$ 0.05 was considered as statistically significant.

\section{Results and Observation}

In this prospective study of 150 patients with ocular symptoms, 75 patients $(50 \%)$ were found to have vitamin-D deficiency with 25-OH vitaminD3 levels below $20 \mathrm{ng} / \mathrm{ml}$ and an equal number of patients had vitamin-D insufficiency (50\%).The maximum distribution of patients was seen in the age group of 51 - 60 years (29\%) [43 patients out of the total 150]. Least percentage of vitamin-D deficient and insufficient was found to be in the age group of 18-20 years $(1.3 \%)$ (Table 1$)$.

The overall male to female percentage was found to be $54 \%$ and $46 \%$ respectively.

Among our study patients there was no significant difference in ophthalmic signs based on age group. There was also no difference in the ophthalmic features in both insufficiency and deficiency.

Dry eye disease (DED) was found in 102 patients (78\%), followed by glaucoma in 33 (27\%), diabetic retinopathy in 30 (22\%), myopia as the common refractive error in 25 patients $(16.7 \%)$, hypertensive retinopathy in 13 out of 150 (8.7\%) and age related macular degeneration (ARMD) in 6 patients (4\%) (Table 2).

Dry eye occurred in 78\% (102 patients: 64 vitamin-D deficient, 38 vitamin-D insufficient). Among this, decreased TBUT (Figure 1a

\begin{tabular}{|c|c|c|c|c|c|}
\hline & & & \multicolumn{2}{|c|}{ Vitamin-D Status } & \multirow{2}{*}{ Total } \\
\hline & & & Deficient & Insufficient & \\
\hline \multirow[t]{14}{*}{ Age } & \multirow{2}{*}{$\begin{array}{c}\text { Upto } 20 \\
\text { years }\end{array}$} & Count & 1 & 1 & 2 \\
\hline & & $\%$ & $1.3 \%$ & $1.3 \%$ & $1.3 \%$ \\
\hline & \multirow{2}{*}{$\begin{array}{c}21-30 \\
\text { years }\end{array}$} & Count & 4 & 4 & 8 \\
\hline & & $\%$ & $5.3 \%$ & $5.3 \%$ & $5.3 \%$ \\
\hline & \multirow{2}{*}{$\begin{array}{c}31-40 \\
\text { years }\end{array}$} & Count & 19 & 10 & 29 \\
\hline & & $\%$ & $25.3 \%$ & $13.3 \%$ & $19.3 \%$ \\
\hline & \multirow{2}{*}{$\begin{array}{l}41-50 \\
\text { years }\end{array}$} & Count & 17 & 18 & 35 \\
\hline & & $\%$ & $22.7 \%$ & $24.0 \%$ & $23.3 \%$ \\
\hline & \multirow{2}{*}{$\begin{array}{c}51-60 \\
\text { years }\end{array}$} & Count & 23 & 20 & 43 \\
\hline & & $\%$ & $30.7 \%$ & $26.7 \%$ & $28.7 \%$ \\
\hline & \multirow{2}{*}{$\begin{array}{c}61-70 \\
\text { years }\end{array}$} & Count & 9 & 16 & 25 \\
\hline & & $\%$ & $12.0 \%$ & $21.3 \%$ & $16.7 \%$ \\
\hline & \multirow{2}{*}{$\begin{array}{l}71-80 \\
\text { years }\end{array}$} & Count & 2 & 6 & 8 \\
\hline & & $\%$ & $2.7 \%$ & $8.0 \%$ & $5.3 \%$ \\
\hline \multicolumn{2}{|r|}{ Total } & Count & 75 & 75 & 150 \\
\hline
\end{tabular}

Table 1: Comparison between age with vitamin-D status.

and $1 \mathrm{~b}$ ) occurred in 30 patients and decreased Schirmer's in 72 patients. This was found to be statistically significant with a p-value of 0.381 . Of those with DED, $62.5 \%$ belonged to the postmenopaus-

\begin{tabular}{|l|c|c|c|}
\hline \multicolumn{1}{|c|}{ Disease } & $\begin{array}{c}\text { Total number } \\
\text { of patients } \\
\text { with ophthal- } \\
\text { mic signs }\end{array}$ & $\begin{array}{c}\text { Vitamin-D } \\
\text { Deficient } \\
\text { (with levels } \\
\text { less than 19 } \\
\text { ng/ml) }\end{array}$ & $\begin{array}{c}\text { Vitamin-D } \\
\text { Insufficient } \\
\text { (with levels } \\
\text { between 20 - } \\
\text { 29 ng/ml) }\end{array}$ \\
\hline Dry eye disease & 102 & 64 & 38 \\
\hline Myopia & 25 & 16 & 9 \\
\hline NPDR & 21 & 11 & 10 \\
\hline PDR & 3 & 1 & 2 \\
\hline CSME & 9 & 6 & 3 \\
\hline $\begin{array}{l}\text { Hypertensive } \\
\text { Retinopathy }\end{array}$ & 13 & 3 & 10 \\
\hline ARMD & 6 & 2 & 4 \\
\hline Disc Suspect & 13 & 3 & 1 \\
\hline $\begin{array}{l}\text { Primary Open } \\
\text { Angle Glaucoma }\end{array}$ & 2 & 1 & 1 \\
\hline $\begin{array}{l}\text { Primary Angle Clo- } \\
\text { sure Glaucoma }\end{array}$ & 11 & & \\
\hline $\begin{array}{l}\text { Ocular Hyperten- } \\
\text { sion }\end{array}$ & & & \\
\hline
\end{tabular}

Table 2: Spectrum of Ophthalmic Manifestations in Vitamin-D deficiency and insufficiency. 
al age group (62.5\%) and 6 belonged to the premenopausal group (37.5\%).

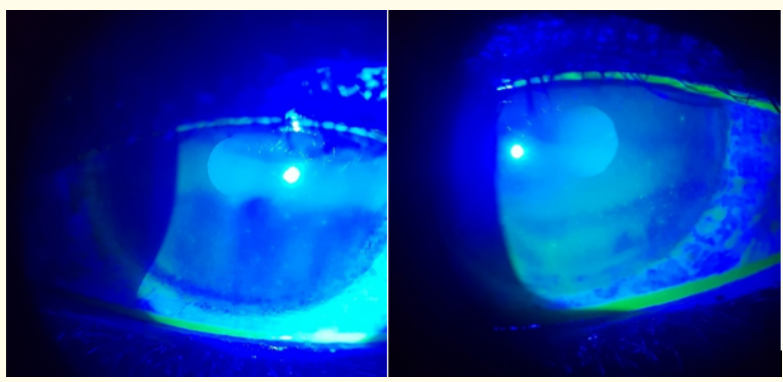

Figure 1a and 1b: Demonstrates decreased TBUT in Dry Eye Disease in patients with Vitamin-D Insufficiency.

A correlation of Vitamin-D status with the different types of glaucoma showed $4(2.7 \%)$ disc suspects, 2 (1.3\%) with primary angle closure and 13 (8.3\%) patients with primary open angle glaucoma. The incidence of glaucoma was higher in women with deficiency and this was found to be statistically significant with $\mathrm{p}$ $=0.03$.

11 patients with low Vitamin-D levels had borderline to high IOP's (ranging from 21 - $30 \mathrm{~mm} \mathrm{Hg}$ ). In them, the relationship between IOP, cup to disc ratios, central corneal thickness and NRR thinning was studied. Pachymetry showed decreased central corneal thinning in 5 patients and NRR thinning clinically and on OCT in 2 patients (Table 3 ).

$52(34.7 \%)$ of the 150 patients had refractive errors. Myopia of varying severity was present in 36 and mixed astigmatism in 16 patients.

\begin{tabular}{|l|c|c|c|}
\hline & $\begin{array}{c}\text { Those with } \\
\text { Vitamin-D } \\
\text { Deficiency }\end{array}$ & $\begin{array}{c}\text { Those with } \\
\text { Vitamin-D } \\
\text { Insufficiency }\end{array}$ & Total \\
\hline Disc suspect & $\begin{array}{c}3 \text { patients } \\
{[4 \%]}\end{array}$ & $\begin{array}{c}1 \text { patient } \\
{[1.3 \%]}\end{array}$ & $\begin{array}{c}4 \text { patients } \\
{[2.7 \%]}\end{array}$ \\
\hline POAG & $\begin{array}{c}5 \text { patients } \\
{[6.7 \%]}\end{array}$ & $\begin{array}{c}8 \text { patients } \\
{[10.7 \%]}\end{array}$ & $\begin{array}{c}13 \text { patients } \\
{[8.7 \%]}\end{array}$ \\
\hline PACG & $\begin{array}{c}1 \text { patient } \\
{[1.3 \%]}\end{array}$ & $\begin{array}{c}1 \text { patient } \\
{[1.3 \%]}\end{array}$ & $\begin{array}{c}2 \text { patients } \\
{[1.3 \%]}\end{array}$ \\
\hline $\begin{array}{l}\text { Ocular Hypertension } \\
\text { was found in a total of } \\
11 \text { patients. }\end{array}$ & & & \\
\hline
\end{tabular}

Table 3: Association of Vitamin-D levels with intraocular pressures.
21 patients (14\%) were found to have non proliferative diabetic retinopathy (NPDR) and 3 patients were found to have advanced proliferative diabetic retinopathy (PDR) with p-value of 1 . Clinically significant macular oedema (CSME) occurred in 9 patients $(6 \%)$ all of whom were treated with grid laser photocoagulation.

Hypertensive retinopathy occurred in 13 (8.7\%,), the most common clinical features being were arteriovenous crossing changes and arteriolar attenuation. ARMD occurred in 6 (4\%) of the total 150 patients. 2 patients had Vitamin-D deficiency and 4 had vitamin-D insufficiency.

Visual loss and visual field defects occurred predominantly in patients with glaucoma due to optic nerve head changes.

\section{Discussion}

In our study population, among the various ocular features, keratoconjunctivitis sicca was the most common manifestation followed by glaucoma. Vitamin-D not only affects calcium homeostasis but plays a role in cell growth and survival along with immune system regulation. Many tissues in the eye activate and respond to vitamin-D, suggesting that vitamin-D is a biologically relevant molecule to study throughout the eye [2].

Lower serum 25-OH vitaminD3 concentrations were associated with a high prevalence of myopia in our patients. This association could be evidence of an underlying biochemical mechanism and loss of homeostasis which can lead to dysregulation of ciliary muscle balance. This explains previous findings that greater time spent outdoors is associated with reduced risk of myopia development [10]. Vitamin-D deficiency in our patients with myopia had a definite higher association with a range between -2 to -6 diopters. This could be due to the regulatory role of vitamin -D in calcium homeostasis [11].

DED prevalence in this study showed that the cause in these patients was due to defective secretion rather than tear film instability and evaporative dry eye.

Poor glycaemic control is a risk factor for the development and progression of diabetic retinopathy, and vitamin-D deficiency has been shown to impair insulin synthesis and secretion [12].

Amongst those with diabetes mellitus and decreased levels of 25-OH vitamin-D3, 21 patients (14\%) had NPDR, 3 patients had advanced PDR. CSME occurred in 9 patients $(6 \%)$ and they were treated with grid laser photocoagulation after which the oedema resolved and vision improved. The anticipation of retinopathy on- 
set is significantly associated with the exaggeration of oxidative stress biomarkers or decrease of antioxidants in African type 2 diabetics, and supplementation with vitamin-D is recommended as complement therapies of type 2 Diabetes Mellitus [13]. Therefore, screening low 25(OH)D3 levels may be a potential simple way for screening diabetic retinopathy among type 2 diabetes.

In our study, hypertensive retinopathy in vitamin-D deficiency and insufficiency was $8.7 \%$. Lower vitamin-D serum levels are associated with the presence of retinal microvascular damage, inflammation, lipid metabolism, and renin-angiotensin-aldosterone system, which are all processes involved in the pathogenesis of arteriosclerosis in hypertensives [14].

ARMD was of the dry type all 6 patients where the fundus showed drusen and RPE atrophy. Inflammation can be a precursor in the pathogenesis of ARMD [15]. Vitamin-D, because of its antiinflammatory and immune-modulating properties, suppresses the cascade of destructive inflammation at the retinal pigment epithelium-choroid interface in early stages of AMD [16].

In patients with vitamin-D deficiency, disc suspects, primary angle closure and primary open angle glaucoma and ocular hypertension were present. We diagnosed ocular hypertension in these patients which could be a precursor of POAG. The most common visual field defect in our patients was nasal step and arcuate scotoma. An early detection with monitoring of visual fields and optic nerve head changes will help to prevent progression and blindness due to glaucomatous disease. The central corneal thickness measured by pachymetry showed thinner central corneal measurements (less than 520 microns) suggesting that an underestimation of IOP during the initial stages can occur in these patients. OCT images showed cupping with NRR thinning (Figure 2).

In glaucoma field studies done earlier, Krefting., et al. [17] had reported that no association exists between vitamin-D levels and intraocular pressure. However, we found that ocular hypertension is a significant association and detection could prevent blindness due to glaucoma. Vitamin-D influences the pathophysiology of glaucoma as a secondary aggravating factor and renders the optic nerve more vulnerable to glaucomatous insult.

\section{Conclusion}

Vitamin-D has varied and multiple protective roles in inflammation, oxidative stress, fibrosis, and angiogenesis. Many tissues in the eye activate and respond to vitamin-D, suggesting that it is a biologically relevant entity to study throughout the eye. Vitamin-D levels and genetic variations influence the development of a wide range of ocular pathologies, such as myopia, age-related macular

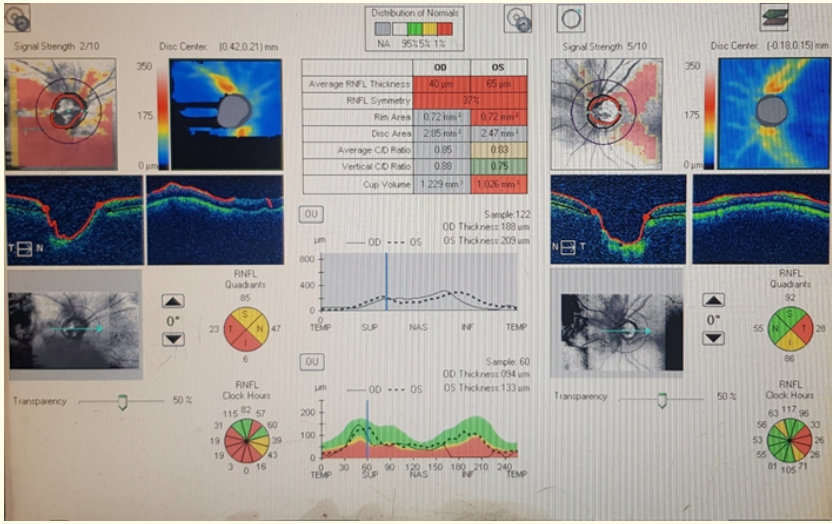

Figure 2: OCT RNFL showing NRR thinning in both eyes in a Vitamin-D deficient patient with borderline intraocular pressures and increased cup-disc ratio.

degeneration, diabetic retinopathy, glaucoma and uveitis.

The therapeutic effect of vitamin-D administration is due to its action at the cellular level reducing inflammatory mediators and enhancing barrier function would plays a protective role in ocular health. Limitation of the study was the that a longer follow up of these patients would have helped to determine the clinical significance of vitamin D supplementation and its effect on progression of ophthalmic features.

\section{Conflict of Interest and Financial Interest} None.

\section{Bibliography}

1. Albert DM., et al. "Calcitriol is a potent inhibitor of retinal neovascularization". Investigative Ophthalmology and Visual Science 48.5 (2007): 2327-2334.

2. Reins RY., et al. "Vitamin D: Implications for ocular disease and therapeutic potential". Experimental Eye Research 134 (2015): 101-110.

3. Johnson JA., et al. "Immuno-localization of the calcitriol receptor, calbinclin-D28k and the plasma membrane calcium pump in the human eye". Current Eye Research 14.2 (1995): 101-108.

4. Alsalem JA., et al. "Characterization of vitamin d production by human ocular barrier cells". Investigative Ophthalmology and Visual Science 55 (2014): 2140-2147.

5. Yin Z., et al. "Vitamin D enhances corneal epithelial barrier function". Investigative Ophthalmology and Visual Science 52 (2011): 7359-7364. 
6. Maya Salomon-Ben Zeev., et al. "Diagnosis of dry eye disease and emerging technologies". Clinical Ophthalmology 8 (2014): 581-590.

7. Marc Pellegrini., et al. "Assessment of Corneal Fluorescein Staining in Different Dry Eye Subtypes Using Digital Image Analysis". Translational Vision Science and Technology 8.6 (2019): 34.

8. Gupta V., et al. "Clinics of ocular tuberculosis". Ocular Immunology and Inflammation 23.1 (2015): 14-24.

9. James G Fujimoto., et al. "Optical Coherence Tomography: An Emerging Technology for Biomedical Imaging and Optical Biopsy". Neoplasia 2.1-2 (2000): 9-25.

10. Jones-Jordan LA., et al. "Visual activity before and after the onset of juvenile myopia". Investigative Ophthalmology and Visual Science 52 (2011): 1841-1850.

11. Imai Y., et al. "Regulation of bone metabolism by nuclear receptors". Molecular and Cellular Endocrinology 310.1-2 (2009): 3-10.

12. Mathieu C., et al. "Vitamin D and diabetes". Diabetologia 48 (2005): 1247-1257.

13. Longo-Mbenza B., et al. "Retinopathy in non-diabetics, diabetic retinopathy and oxidative stress: A new phenotype in Central Africa". International Journal of Ophthalmology 7 (2014): 293301.

14. Unal Mutlu., et al. "Vitamin-D and retinal microvascular damage: The Rotterdam Study". Medicine (Baltimore) 95.49 (2016): e5477.

15. Millen AE., et al. "Vitamin-D status and early age-related macular degeneration in postmenopausal women". Archives of ophthalmology 129.4 (2011): 481-489.

16. Hageman GS., et al. "An integrated hypothesis that considers drusen as biomarkers of immune mediated processes at the RPE-Bruch's membrane interface in aging and age-related macular degeneration". Progress in Retinal and Eye Research 20.6 (2001): 705-732.

17. Krefting EA., et al. "Vitamin D and intraocular pressure: results from a case-control and an intervention study". Acta Ophthalmologica 92 (2014): 345-349.

\section{Assets from publication with us}

- Prompt Acknowledgement after receiving the article

- Thorough Double blinded peer review

- Rapid Publication

- Issue of Publication Certificate

- High visibility of your Published work

Website: www.actascientific.com/

Submit Article: www.actascientific.com/submission.php Email us: editor@actascientific.com

Contact us: +919182824667 